\title{
A review of methods and algorithms for optimizing construction scheduling
}

\author{
J Zhou $^{1 *}$, PED Love ${ }^{1}, \mathrm{X}_{\text {Wang }}{ }^{1}, \mathrm{KL} \mathrm{Teo}^{1}$ and Z Irani ${ }^{2}$ \\ ${ }^{1}$ Curtin University, Perth, Australia; and ${ }^{2}$ Brunel University, Middlesex, UK
}

Optimizing construction project scheduling has received a considerable amount of attention over the past 20 years. As a result, a plethora of methods and algorithms have been developed to address specific scenarios or problems. A review of the methods and algorithms that have been developed to examine the area of construction schedule optimization (CSO) is undertaken. The developed algorithms for solving the CSO problem can be classified into three methods: mathematical, heuristic and metaheuristic. The application of these methods to various scheduling problems is discussed and implications for future research are identified.

Journal of the Operational Research Society (2013) 64, 1091-1105. doi:10.1057/jors.2012.174

Published online 13 March 2013

Keywords: algorithm; construction; optimization; scheduling

\section{Introduction}

Scheduling the execution processes for a construction project is a complex and challenging task (König and Beißert, 2009). The selection of resources (eg, labour, plant and equipment) is the most important part of scheduling and should be considered incongruence with site restrictions and the work to be undertaken (Jaśkowski and Sobotka, 2006). As projects are unique in nature, the creation of a schedule for construction tasks by a planner, for example, should consider an array of conditions such as technological and organizational methods and constraints, as well as the availability of resource to ensure that a client's needs and requirements in terms of time, cost and quality are met (Jaśkowski and Sobotka, 2006).

Construction project scheduling has received a considerable amount of attention over the last 20 years (eg, Shanmuganayagam, 1989; Adeli and Karim, 1997; Hegazy, 1999a; Zhang et al, 2006d; Fang, 2012). A plethora of methods and algorithms have been developed to address specific scenarios or problems, particularly significant practical issues such as:

- scheduling with uncertain estimates on activity durations;

- integrated planning and scheduling and resource allocation; and

- scheduling in unstructured or poorly formulated circumstances.

\footnotetext{
${ }^{*}$ Correspondence: $J$ Zhou, School of Built Environment, Curtin University, GPO Box U1987, Bentley, Perth, WA 6102, Australia.

E-mail: zhouhit@gmail.com
}

Fundamentally, the construction schedule optimization (CSO) problem is a subdivision of the project scheduling optimization problem. Many techniques and algorithms used for solving the project scheduling problem can be directly applied to the CSO problems. A detailed review of the project scheduling can be found in Willis (1986), Icmeli et al (1993), Ozdamar and Ulusoy (1995), Herroelen (2005), Lancaster and Ozbayrak (2007) and Hartmann and Briskorn (2010). While, different projects have varying features, so does the CSO problem. Construction projects are unique in nature and each has their own site characteristics, weather condition, and crew of labour and fleet of equipment. As a result, it is difficult to accurately predict the exact duration of each activity. The CSO problem involves the scheduling of construction activities subjected to precedence and/or resource constraints. The aim of the CSO is to determine a feasible schedule of these activities to achieve certain predefined objective, for example, the shortest project duration, lowest cost or highest profit subject to the problem constraints. Against this contextual backdrop, this paper provides a review of techniques and algorithms that have been developed to address the CSO problem.

\section{Construction scheduling problems}

Construction scheduling is concerned with optimally sequencing activities over time and allocating resources accordingly. The duration criterion is a critical factor taken into account by a client when evaluating and selecting bids with the project deadline normally being pre-specified. 
Contractors always strive to minimize the project duration so as to obtain an advantage during a bid's evaluation. For example, they may 'crash' a project's duration (ie, the shortest possible time for which an activity can be scheduled) by allocating more resources (if sufficient resources are available) to expedite construction activities. However, crashing a project's duration invariably increases the cost, as additional resources are required. This is due to the interdependency that exists between time and cost. For example, compressing a project's duration will lead to an increase in direct costs (plant and equipment, materials and labour cost) and a decrease in indirect costs (project overhead), and vice versa (Kasprowicz, 1994; Laptali et al, 1997; Nkasu and Leung, 1997; Wang and Huang, 1998).

To be successful in a bid evaluation, cost is also a factor that should be considered by a planner. During the construction phase, the cost for material, plant and equipment and labour are classified as direct costs, while insurances and taxes, for example, are indirect costs. Typically clients, particularly developers, aim to minimize project cost and duration in order to reduce their cost of finance and maximize their return on capital. For contractors, minimizing cost increases their profit and an earlier project completion reduces the risk of inflation and labour shortage (Ng and Zhang, 2008). Project scheduling should therefore consider time and cost simultaneously as a 'trade-off' exists. Thus, the original single objective optimization problem (ie, optimal time or cost) is shifted to a bi-objective optimization problem (ie, optimal time-cost). The construction time-cost optimization problem has been examined extensively in the construction engineering and management literature (eg, Siemens, 1971; Tamimi and Diekmann, 1988; Que, 2002; Rogalska et al, 2008; Wu et al, 2009; Yu et al, 2010; Wongwai and Malaikrisanachalee, 2011).

'Crash duration' is a commonly used method to expedite the construction process. If a client, for example, requires their project to be completed earlier, a contractor may provide additional resources to shorten the duration of designated activities. As previously noted, for this to occur, resources need to be readily available. In practice, this assumption is often deemed to be unrealistic, as construction projects are subjected to constraints that play a key role in determining their schedule, for example, activity dependency, limited working area, information availability (Sriprasert and Dawood, 2003).

Activity dependency, time, cost and resources are the constraints normally considered when scheduling under the auspices of traditional project management. Solutions to this optimal scheduling problem with or without consideration of these constraints vary (Toklu, 2002). Activity dependency or precedence relationship is the most basic constraint that exists in construction projects. In a construction process, an activity cannot start until all its precedence activities are completed. In addition, the start time of each activity cannot be later than its latest start time in order to finish the project within the demanded duration (Guo et al, 2010). The working space is always limited in a construction project. A working area may be required by several different activities at the same time. Therefore, determining how to optimally manage a working area to facilitate activity scheduling will directly affect project performance. Such a situation is called a 'space-time' conflict problem between construction site activities (Kazi et al, 2009). Resources are the most influential constraints in construction, as they determine the feasibility of a project schedule and whether it is optimal (Chen and Weng, 2009). Schedule reduction is heavily dependent on the availability of resources. Information constraints, which consist of drawings, specifications, safety and risk assessments, authorizations to work, also have significant impact on the construction scheduling problem. Information flow between activities, for example, has often been overlooked (Sriprasert and Dawood, 2002). A detailed review of constraints influencing construction can be found in Sriprasert and Dawood (2002), Zhang et al (2006a), Zhang et al (2006b), Kim and Ellis (2008), Wu et al (2009) and Liu and Wang (2010).

Another 'trade-off' from 'crash' duration is its influence on project quality ( $\mathrm{Li}$ et al, 2000). Thus, the time-cost bi-objective optimization problem could be expanded into a time-cost-quality multi-objective optimization problem, that is, minimizing the construction time and cost while maximizing the quality. To involve quality as an additional objective requires the quality to be quantifiable. In doing so, the following major challenges arise (El-Rayes and Kandil, 2005):

- the difficulty in measuring and quantifying the impact of each resource utilization option on the quality of the activity being considered; and

- the complexity of cumulating quality performance at the activity level to an overall quality at the project level.

In addressing the above challenges, El-Rayes and Kandil (2005) proposed a quality objective function that consists of a number of measurable quality indicators for each activity. It also comprises two types of weights that are used to estimate the overall quality performance at the project level, that is, weight of quality indicator compared with other indicators in activity and weight of activity compared with other activities in the project (El-Rayes and Kandil, 2005). Therefore, the traditional two-dimensional time-cost trade-off problem is transformed into a threedimensional time-cost-quality trade-off problem. The proposed method could provide useful information for the decision maker to make trade-off decisions especially in a high quality demanded environment. Research including quality as an additional objective has been limited to date. 


\section{Solving CSO problems}

A plethora of methods have been propagated to address the CSO problem, which can be classified as: mathematical, heuristic and metaheuristic. Each of the methods are examined and discussed below. A classification of the research is shown in Table 1.

To implement the mathematical method, the problem needs to be explicitly formulated (ie, the objective function and constraints). This is a time-consuming task and difficult for construction planners who do not have the specified mathematical knowledge and background. Some mathematical searching algorithms, for example hill climbing algorithm, are single objective oriented and likely to be trapped into local optimality. Therefore, methodologies, by which a global optimality can be obtained, are highly demanding such that an optimal schedule can be achieved. Constraints are critical factors for solving the CSO problem. Traditional mathematical methods usually treat constraints and objective function separately, that is, optimizing the objective function subject to the constraints. To solve such a problem, a feasible point to initiate the searching process and algorithms that can guarantee that the constraints are satisfied is needed.

\section{Mathematical methods}

Critical path method. The critical path method (CPM) is a widely used project scheduling algorithm that was developed in the late 1950s (Kelley and Walker, 1959; Kelley, 1961). It can be applied to any project with interdependent activities, such as construction, aerospace engineering, software development, industrial manufacturing. To date, CPM is the most commonly used scheduling tool in the construction industry. Fundamentally, however, CPM can only deal with optimization problems with a single objective. CPM is commonly used in conjunction with the Programme Evaluation and Review Technique (PERT).

The conventional CPM was developed to analyse the project network logic diagram. The essential technique to implement CPM is to construct a model of the project that involves the following items (Samuel, 2010):

- a list of all activities within the project;

- the duration of each activity; and

- the precedence relationship between the activities.

With the above information, CPM can be used to calculate the longest path (critical path) to complete the project, and the earliest and latest starting and finishing time of each activity without delaying the completion of the project. Activities on the critical path are termed as 'critical activities' and those not on the critical path are 'float activities'. Figure 1 provides an example of a CPM network diagram with seven activities on nodes. In
Figure 1, there is only one critical path, that is, S-A-B-C-E. Therefore, A, B and $\mathrm{C}$ are critical activities. Any delay to these activities will delay the entire project. D, F, G and $\mathrm{H}$ are float activities, which can be delayed without influencing the project's duration. Therefore, CPM can be used in this instance to determine the shortest possible time to complete the project. A detailed review of how CPM has been used in the construction industry can be found in Galloway (2006).

A major limitation of CPM schedules is their reliance on time and dependency constraints. In addressing this limitation, a two-stage approach to resource constraints representation has been developed. In the first stage, the precedence relationships are defined, while in the second stage resources are introduced in the scheduling using resource allocation or leveling algorithms (Antill and Woodhead, 1982; Moder et al, 1983; Cormican, 1985; Tamimi and Diekmann, 1988). Stevens (1990) suggest that resources should be ignored during the first stage. However, Waugh and Froese (1991) have argued that realistic construction activities cannot be developed without considering resources. Moreover, it is often difficult to determine the logic between technological and resources constraints (Barrie and Paulson, 1984). To overcome such difficulties, Waugh and Froese (1991) proposed an approach, called 'A Construction Planner' (ACP), which explicitly accounts for all constraints simultaneously including resource constraints using a single stage approach. The ACP provides a more robust model of planning that took advantage of advanced computer technologies (Waugh and Froese, 1991).

Liu and Li (2003) developed a method to accommodate resource constraints and repetitive scheduling known as the Resource-Activity Critical-Path Method. On the basis of the resource-technology combined precedence relationships, the start/finish times and the floats are defined as resource-activity attributes. However, minimization of the overall project cost was not considered in this approach. In addressing this issue, Kallantzis and Lambropoulos (2004) present a scheduling method for determining the critical path in linear projects, which takes into account maximum time and distance constraints in addition to the commonly used minimum time and distance constraints. The proposed method incorporates the maximum constraints into the schedule and all linear activities are grouped into four categories according to their critical status and the abilities to influence the project duration. In this method, the production rates are assumed to be fixed and are unable to deal with uncertain resource availabilities.

\section{Integer programming $(I P)$, linear programming $(L P)$ and IP/LP algorithms}

A number of analytical algorithms have been applied to address the CSO problems, such as IP, LP and hybrid 
Table 1 Classification of CSO research 


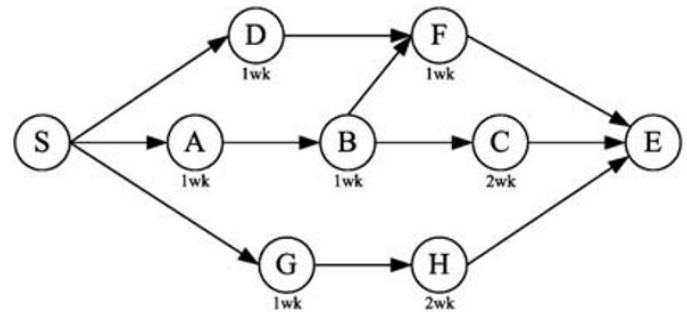

Figure 1 Example of a CPM network.

IP/LP algorithm. LP is a mathematical method for solving the optimization problem with linear objective functions subject to linear equality and inequality constraints (Kantorovich, 1940). LP problem can be expressed in the following general form:

$$
\text { Minimize } \mathbf{c}^{T} \mathbf{x}
$$

Subject to

$$
\begin{gathered}
A x \leqslant b \\
\mathbf{x} \geqslant 0
\end{gathered}
$$

where $\mathbf{x} \in \mathbb{R}^{n}$ is the unknown variable vector, $\mathbf{A} \in \mathbb{R}^{m \times n}$ is the coefficient matrix and $\mathbf{b} \in \mathbb{R}^{m}, \mathbf{c} \in \mathbb{R}^{n}$ are coefficient vectors. The objective of the problem is to optimize (maximize or minimize) the linear objective function subject to constraints (2) and (3). If some or all of the variables are restricted to be integers, then, the LP problem is transformed into an IP problem as follows:

$$
\text { Minimize } c^{T} x
$$

Subject to

$$
\begin{aligned}
& A x \leqslant b \\
& \mathbf{x} \geqslant 0
\end{aligned}
$$

$x_{i}$ integer for some or all $i=1,2, \ldots, n$

For example, a time-cost trade-off construction scheduling problem presented by LP model can be expressed as follows (Liu et al, 1995):

$$
\operatorname{Minimize} \sum_{i=1}^{n} C_{i}
$$

Subject to

$$
\begin{gathered}
S_{i} \geqslant 240, i=1,2, \ldots, n \\
S_{i}+D_{i} \leqslant 24 D_{\max }, i=1,2, \ldots, n \\
S_{a}+D_{a} \leqslant 24 S_{b}, \text { for each precedence } a \rightarrow b \\
C_{i} \geqslant 24 M_{i j} D_{i}+B_{i j}, i=1,2, \ldots, n, j=1,2, \ldots, O_{i}
\end{gathered}
$$

$$
\begin{gathered}
C_{i} \geqslant 24 C_{i}^{\min }, i=1,2, \ldots, n \\
D_{i} \geqslant 24 D_{i}^{\min }, \quad i=1,2, \ldots, n
\end{gathered}
$$

where $C_{i}$ is the cost of activity $i ; S_{i}, D_{i}$ and $O_{i}$ are the start time, duration and number of inequality constraints of activity $i$, respectively. $D_{\max }$ is the maximum allowable overall project duration. $C_{i}^{\mathrm{min}}$ and $D_{i}^{\mathrm{min}}$ are the minimum cost and duration of activity $i$, respectively. $M_{i j}$ represents the slop of inequality constraint connecting the adjacent active options pair. $n$ denotes the total number of all the activities. $B_{i j}$ is the intercept of cost for option $j$ with respect to activity $i$.

The objective of this problem is to minimize the overall project cost subject to constraints (9)-(14). Some typical methods, such as the simplex algorithm, Criss-cross algorithm and interior point methods can be utilized to solve the LP problem efficiently. A similar problem, represented by IP model, can be expressed as (Liu et al, 1995):

$$
\operatorname{Minimize} \sum_{i=1}^{n} C_{i}
$$

Subject to

$$
\begin{gathered}
S_{i} \geqslant 240, i=1,2, \ldots, n \\
S_{i}+D_{i} \leqslant 24 D_{\max }, i=1,2, \ldots, n \\
S_{a}+D_{a} \leqslant 24 S_{b}, \text { for each precedence } a \rightarrow b \\
\sum_{j=1}^{O_{i}} C_{i j} X_{i j}=C_{i}, i=1,2, \ldots, n \\
\sum_{j=1}^{O_{i}} D_{i j} X_{i j}=D_{i}, i=1,2, \ldots, n \\
\sum_{i=1}^{O_{i}} X_{i j}=1, i=1,2, \ldots, n \\
X_{i j} \in\{0,1\}, i=1,2, \ldots, n, j=1,2, \ldots, O_{i}
\end{gathered}
$$

where $X_{i j}$ are decision variables assigned to option $j$, activity $i$. Here, the objective is to minimize the overall project cost subject to constraints (16)-(22). The decision variables $X_{i j}$ are restricted to be integers chosen between 0 and 1. Constraints (21) and (22) ensure that only one option is chosen for each activity during the optimization process. Several efficient approaches for solving the IP problems have been developed such as cutting-plane method, branch and bound method, branch and cut method, and branch and price method (Chen et al, 2010). For more information of IP and LP, refer to Beasley (1996), Karlof (2005) and Jünger et al (2010).

Mathematical methods for scheduling have received a considerable amount of attention due to their innate 
efficiency and accuracy. Meyer and Shaffer (1963) applied IP to solve the linear and discrete relationship of different activities within a scheduling optimization problem in a highway construction project that had a number of repetitive activities. Similarly, Mattila and Abraham (1998) developed an integer LP approach to solve the highway construction project using the resource leveling technique. The concepts of rate and activity float are introduced based on the resource utilization on a particular activity. If activities have common resources, then rate float can be used to achieve better resource utilization (Mattila and Abraham, 1998). A disadvantage of this method is that the computational burden may grow tremendously as the problem size increases. In addition, this method has a single focus is single objective focus (ie, leveling the resources) and thus the maximization of production rates is not considered.

Elazouni and Gab-Allah (2004) proposed an IP finance-based scheduling method to produce feasible schedules that balance the financing requirements of activities at any period with the cash available during that same period. The proposed method can be used to minimize total project duration and fulfil finance availability constraints (Elazouni and Gab-Allah, 2004). Besides IP, LP is also applied in construction scheduling area especially for solving those problems with linear objective functions and constraints (Gomar et al, 2002).

To overcome the single objective oriented limitation of traditional scheduling methods, such as CPM, Ipsilandis (2007) proposed a multi-objective LP model for scheduling linear repetitive projects that considers cost elements regarding the project's duration, the idle time of resources and the delivery time of the project's units. The proposed model is used to generate alternative schedules based on the relative magnitude and importance of the different cost elements (Ipsilandis, 2007). The LP range sensitivity analysis can provide useful information regarding cost trade-offs between project and resource delays. It can provide managers with the capability to consider alternative schedules besides those defined by minimum duration or minimum resource work-breaks (Ipsilandis, 2006). It is suggested that weights could be introduced into the multi-objective function so as to enhance the performance of the proposed method. Huang and Halpin (2000) developed a graphically based approach to assist in the LP of linear scheduling analysis, which they referred to as the Planning and Optimization for Linear Operations system. This system provides a graphic LP modelling environment in which model formulation can be accomplished in a graphic and interactive fashion. However, the solutions obtained are not guaranteed to be cost optimal. In addition, the method is currently only applicable to repetitive activities, that is, those one-off activities need to be dealt with separately.
Burns et al (1996) propagated a hybrid optimization approach that integrates LP and IP for determining the time-cost trade-off solution of a construction scheduling problem. The method is applied in two stages: (1) using LP to generate a lower bound of the minimum direct curve; and (2) using IP to find the exact solutions. The proposed hybrid LP/IP method provides construction planners with an efficient way of analysing the time-cost trade-off problem.

Dynamic programming. Dynamic programming is a mathematical method applicable for solving complex problems that can be broken down into some subproblems. It is efficient for solving those problems with overlapping sub-problems (Dasgupta et al, 2006). Numerous examples of dynamic programming can be found in the construction engineering and management literature. For example, Robinson (1975) presented a dynamic programming approach to solve time-cost trade-off problems. Moselhi and El-Rayes (1993) proposed a dynamic programming model by introducing a cost variable into the optimization process. The model performs the solution with two stages:

1. Forward process, which involves a time-cost trade-off analysis to determine local minimum conditions is employed;

2. Backward process, which involves simple scanning and selecting process that ensures an overall minimum state is attained.

Formulating objective functions and constraints is a time-consuming and arduous task. Few construction planners are trained to obtain the required mathematical knowledge to perform such a formation and as such its application to construction and engineering project scheduling has been limited to date.

\section{Heuristic methods}

Heuristic methods are based on the past experience for problem solving. Prevalent heuristic methods include Fondahl's (1961), Structural model (Prager, 1963), Siemens approximation (Siemens, 1971) and structural stiffness (Moselhi, 1993).

Fondahl (1961) developed a precedence methodology as an alternative to CPM. The method provides an effective manual process to determine a schedule instead of using a computer-based CPM. In Fondahl's approach, a 'circle and connecting line' diagram derived from process flow diagrams or flow-charts was used to address a number of issues such as the time-cost trade-off problem (Fondahl, 1961). Noteworthy, current project management software utilizes the manual calculation approach developed by Fondahl 
On the basis of Fulkerson's (1961) method, Prager (1963) proposed a structure model to interpret the network flow formulation. The activities of a project and the progress towards its completion are described as jobs and events, respectively. Each job is represented by a structural member that consists of a rigid sleeve containing a compressible rod with a piston at its protruding end. The events are arranged between the jobs represented by thin rigid discs. Then, an algorithm is provided for the scheduling calculation. It is assumed that the normal and crash completion times are known for each job associating with linearly varied cost of completing the job between these times. A more complicate non-linear relationship between time and cost is not considered.

Siemens (1971) developed an algorithm that can effectively reduce the duration of a project when its expected duration exceeds a predetermined limit. The algorithm can shorten the project duration at minimum cost by determining which activity to expedite and by what amount. The proposed algorithm is simpler than some analytic methods (eg, LP), as it can be calculated and applied to time-cost trade-off problems without the use of a computer. However, the solution obtained by this algorithm cannot be guaranteed to be optimal. In fact, it is difficult to determine whether the obtained solution is optimal or not.

Moselhi (1993) proposed a method for CPM scheduling that optimizes the project duration to minimize total cost. The method can be used to produce constrained scheduling that accommodates contractual completion dates of projects. The proposed method is based on the well-known 'direct stiffness method' for structural analysis, which establishes a complete analogy between the structural analysis problem with imposed support settlement and that of project scheduling with imposed target completion date. The CPM network is replaced by an equivalent structure whose compression is equivalent to the project schedule compression. The cost required to achieve such a compression is represented by the sum of all member forces (Moselhi, 1993).

Zhang et al (2006a) developed a heuristic method for scheduling the multiple-mode repetitive construction project subject to resource constraints. The method categorizes activities according to possible combinations into groups and schedules all the activities in the selected group simultaneously to minimize the project duration. A permutation tree-based procedure is employed to determine the alternative activity combinations. The heuristic algorithm ranks all alternative combinations of activities and selects the one leading to a minimal increase in project duration. A framework of the project scheduling system is constructed so as to implement the heuristic method (Zhang et al, 2006a). Minimizing the project duration may reduce the indirect cost; however, it may also increase the direct cost or the overall cost that was not considered in this method.
Elazouni (2009) proposed a heuristic method for scheduling multiple projects subject to cash constraints. The method determines cash availability during a given period and identifies the schedules for all possible activities as well as the cash requirements for each schedule. The schedules are evaluated according to their impact on the project's duration. The influence of the activities on cash flow within the selected schedule is also determined. Comparison between the proposed approach and IP on a project with 15 networks and 60 activities shows that the solutions obtained using the proposed heuristic method are comparable to the optimum solutions (Elazouni, 2009). This heuristic method can be easily integrated into management software to handle the project scheduling problem subject to finance constrained conditions (Elazouni, 2009). However, a drawback of this method is that the computation effort grows exponentially as the number of eligible activities and the time span increase.

Hegazy et al (2000) modified the heuristic resourcescheduling solutions by introducing multi-skilled resources. The developed approach stores and utilizes information about the resource that can be substituted. Using this information, less utilized resources can be combined to substitute the constrained resources during the shortage period in order to reduce the project cost and time. To improve the resource substitution approach, Wongwai and Malaikrisanachalee (2011) introduced an alternative heuristic for multi-skilled resource scheduling problem. An augmented resource substitution rule and resource-driven task duration are presented to increase the starting opportunity for activities. The limitation of the proposed method is that some other real-world resource substitution alternatives are not considered such as working overhead or temporary external workers. The method is only valid for start-to-finish relationships. In addition, minimization of the overall project cost is not considered in the aforementioned methods.

Heuristic methods are non-computer approaches that require less computational effort than mathematical methods and can invariably be calculated using manual means. Owing to their simplicity, heuristic methods have been widely adopted to solve the CSO. However, since traditional heuristic methods can only optimize one objective, a global optimum is not guaranteed. Heuristic methods do not provide a pool of the possible solutions from which the construction planner may choose a suitable solution according to different construction scenarios. Being inefficient for solving the multi-objective scheduling problems poses a difficulty for their further applications. Heuristic methods are problem dependent and therefore cannot be generalized to all other cases.

\section{Metaheuristic method}

Metaheuristic methods are used for solving combinatorial optimization problems whose optimal solution is over a 
discrete search space. The metaheuristic method can improve a candidate solution by iterative computation with regard to a given criterion without making too many assumptions about the problem at hand. Popular metaheuristic methods used are naturally inspired methods that have been developed from natural behaviours. The purpose of using naturally inspired methods (eg, genetic algorithm (GA), ant colony optimization (ACO) and particle swarm optimization (PSO)) is to mimic natural processes to ensure optimal solutions. Among these methods, the GA has become the most popularly used approach for addressing the CSP in the construction and engineering literature.

Genetic algorithm. GA belongs to the larger class of evolutionary algorithms (EA) that solves optimization problems using techniques based on natural evolution. Other members of EA include genetic programming, evolutionary programming and evolution strategy. A GA is a random searching algorithm based on the mechanism of natural selection and survival of the fittest. The three most important phases involved in GA are selection, crossover and mutation (Figure 2). To utilize GA, all the decision variables, for example options for each construction activities, are encoded into a string called a chromosome whose genes are represented by binary digits, integers or real numbers. Then, an initial population is chosen randomly and each chromosome's fitness is evaluated with regard to the objective function. According to the fitness, a selection method is employed and a candidate population is created for crossover that allows information exchanges between parents to generate new offspring. In the mutation phase, genes are altered on some randomly chosen locus to eliminate the premature problem caused in the crossover. Then, a new population is generated for the next round iteration. The GA is an efficient global parallel searching algorithm that can accumulate information from the searching space and obtain an optimal or suboptimal solution adaptively.

In complex projects, resource allocation and leveling are invariably dealt with as two distinct sub-problems solved predominately using heuristics that cannot guarantee optimum solutions. Chan et al (1996) used a GA-scheduler to deal with construction resource scheduling problem. The proposed GA-scheduler is capable of resource leveling and limited resource allocation without using any heuristic rules. Two types of constraints were considered: hard and soft. The hard constraints cannot be violated or relaxed.

The soft constraints can be relaxed to some extent with a penalty on the performance. The GA-scheduler can obtain solutions that have at least the same project duration or even shorter duration than those solutions generated by heuristic methods. Moreover, computation effort of using the GA-scheduler does not exponentially increase. Chan et al (1996) also recommended the use of local optimization or greedy algorithm for a quick search of the vicinity of the GA-produced solution to locate the nearby optimum.

Due to the insufficiency of the heuristic and mathematical programming methods for solving the large scale CPM network problems, Feng et al (1997) employed a GA to solve construction time-cost trade-off problem using Pareto Optimality. For a multi-objective optimization problem, Pareto optimality denotes the situation that no further improvement can be made to an individual objective without sacrificing at least one of the other objectives. Pareto Front is a set of solutions satisfying the conditions of Pareto optimality based on which the designers could make trade-off decisions. Using GA and Pareto Front, they proposed an algorithm for solving the construction time-cost trade-off problem. The algorithm shows its efficiency by searching only a small fraction of the total searching space (Feng et al, 1997). However, the method is only applicable to the finish-to-start relationship within the activities, and it is also unable to deal with limited resources.

Leu and Yang (1999) suggested a GA-based multicriteria computational optimal model for the CSO that integrates the time-cost trade-off, resource limit and resource leveling models. To overcome the computational inefficiency due to repair, a new crossover operator, UX3,

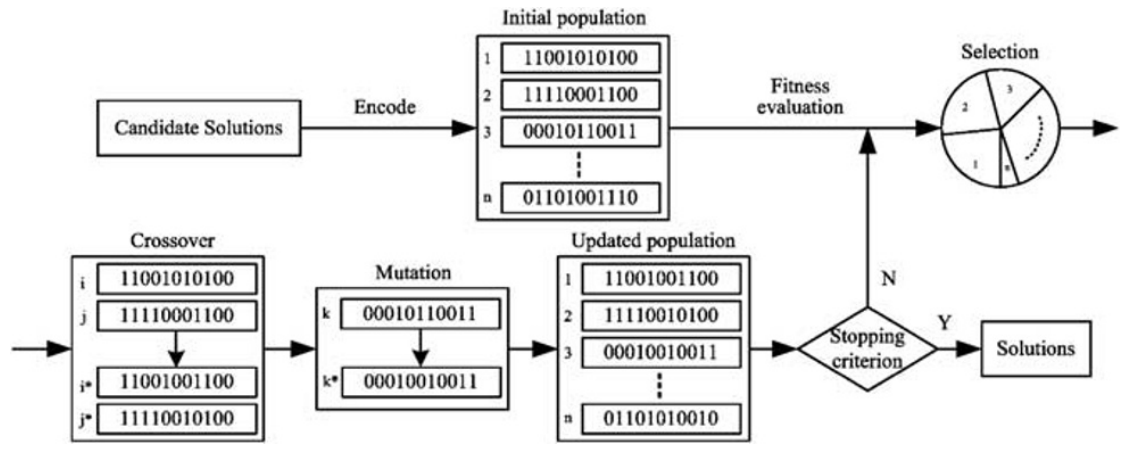

Figure 2 Operation of GA. 
is developed, which takes into account activity precedence relationships when writing characters from substrings into offspring strings. A multiple attribute decision-making method is employed to find the non-dominated solutions.

To reduce the computational effort involved by using a GA, Li and Love (1997) proposed an improved GA for facility time-cost scheduling optimization. An improved crossover operator was introduced to ensure that the offsprings are feasible solutions. An improved mutation operation was also introduced to adjust the crashing time such that the constraints are met. A limitation of the method proposed by $\mathrm{Li}$ and Love (1997) is that crash times are treated as continuous variables, which can be impractical. Furthermore, Li and Love (1997) did not consider resource constrained situations.

In addressing the shortcomings of $\mathrm{Li}$ and Love (1997), Hegazy (1999a) developed an approach that integrated GA and the commercial scheduling software Microsoft Project 4.1 to deal with construction time-cost trade-off scheduling problem. Using the CPM engine and other functions such as resource leveling embedded in the software, resource availability is considered during the evolutionary computation process. The developed method considers project deadline, daily incentive, daily liquidated damages, and daily indirect cost into its formulation and uses total cost as the objective function. Due to its random nature, a considerable amount of computation time is required for large network problems. Li et al (1999) stated that the traditional GA-based system for solving time-cost trade-off problems suffers from the following limitations as the:

- objective function is formulated manually based on the time-cost curves; and

- system only deal linear time-cost relationship.

To overcome these limitations, Li et al (1999) developed a computation method integrating machine learning and GA by which a quadratic template is introduced to capture the non-linearity of time-cost relationships. A quadratic time-cost curve is generated from historical data and used to formulate the objective function that can be solved by the GA. Improved crossover and mutation operators were also used to enhance the computation speed. In $\mathrm{Li}$ et al (1999), a quadratic time-cost relationship was considered, though this may not be appropriate in more complex projects. Further improvement should be made to interpret the non-linear relationship between time and cost.

Hegazy (1999b) used GA to search near-optimum solution for the resources allocation and leveling problems simultaneously. Random priorities were introduced into selected tasks and their impact on the schedule is monitored. In this instance, GA is able to search for an optimal set of tasks' priorities that produces shorter project duration and better-leveled resource profiles. A major advantage of the method is its simplicity and as a result can be integrated into commercial project management software.

Senouci and Eldin (2004) presented an augmented Lagrangian GA model for construction resource scheduling problem. The proposed model considers several issues such as precedence relationships, multiple crew strategies, total project cost minimization and time-cost trade-off. In this model, resource leveling and resource-constrained scheduling problems are performed simultaneously. By taking consideration of continuous linear and non-linear cost-duration curves and resource-duration curves, an objective function, minimizing the total project cost, was formulated subject to several constraints. Then, a quadratic penalty function was used to transform the resource scheduling problem to an unconstrained problem to facilitate the application of GA, which could yield optimal or suboptimal solutions.

Sriprasert and Dawood (2003) used a GA to solve construction scheduling problem considering the following constraints physical, contract, resource and information. The proposed GA approach can alter task's priorities and construction method so as to achieve optimal or suboptimal solution. Microsoft Project software was chosen to implement the GA system and interfaces were developed using Visual Basic for Applications language. The study considered multi-objectives (ie, duration, cost, and resource and space utilization), which can be optimized simultaneously by using multi-objective weighting method. This is a useful and simple method to deal with multi-objective problems. However, since the weights are predetermined, system performance can be affected by those dominant objects. Choosing appropriate weights pose difficulties for the applications of this method.

Zheng et al (2004) developed a multi-objective model for construction time-cost optimization problems. The feature of the method is to solve the trade-off problem between time and cost so as to minimize them simultaneously. For dealing with the multi-objective problem, a modified adaptive weight approach (MAWA) was proposed that can adjust the scope of the next search according to the performance of the current population in obtaining an optimum (Zheng et al, 2004). A new fitness function was also proposed in accordance with the proposed MAWA. The modified adaptive weights can guide the algorithm to search a larger space and increase the diversity of exploration. To overcome the weakness of the roulette wheel' selection method used by traditional GA, Zheng et al (2005) employed a Pareto ranking approach for the selection phase of the GA. With Pareto ranking, all nondominated solutions in current population are grouped and ranked. The group with the higher rank will have a greater chance to survive. It ensures equal reproductive probability among non-dominated solutions on the same level (Zheng et al, 2005). A Niche formation technique was also 
introduced to enhance the population diversity. In this approach, the resources are assumed to be unlimited with a condition that an extra amount can only be obtained at a higher price, which, however, may be impractical in practice.

El-Rayes and Kandil (2005) proposed a GA-based approach to solve a highway construction scheduling problem. A new objective, 'quality', was introduced that transformed the traditional time-cost trade-off problem to a time-cost-quality trade-off problem. The objective of the optimization problem is to minimize construction time and cost, while maximize its quality. A number of measurable quality indicators for each activity in the project were introduced in order to quantify the construction quality. Pareto optimality and Niche comparison rule were introduced for GA computation.

Long and Ohsato (2009) developed a GA-based method for scheduling repetitive construction projects considering project duration, cost or both of them. Resource constraints, different attributes activities and different relationship between direct cost and duration were considered. Unlike the previous research, which aimed to balance the cost of delay with the cost of discontinuities, Long and Ohsato (2009) extended the repetitive CSO to a non-linear and complex optimization problem by presenting a nonlinear combined performance index according to the deviations from the minimum cost and duration. A twostage sub-procedure (SP1) was employed to evaluate the fitness function for the GA computation. The proposed approach could assist the planners to make alternative resource selection decisions to minimize project duration and cost. However, Long and Ohsato (2009) did not consider that several crews can work simultaneously and therefore only deal with deterministic information.

In practice, the actual time and cost for each activity in construction option may be uncertain. This would make it very difficult for the managers to make a decision. To overcome this difficulty, Eshtehardian et al (2008) proposed an optimization approach dealing with construction time-cost trade-off problem with uncertainties. Fuzzy theory was introduced by which the project time and cost are assigned with fuzzy numbers. Then, GA was applied to solve the optimization problem. The proposed model has the ability to adapt to deterministic and uncertain environment by using the $\alpha$ cut method. New nondominated solutions can be obtained using the $\alpha$ cut property according to the project manager's acceptance of risk level.

Kim and Ellis (2008) used a permutation-based elitist GA to solve large-scale resource constrained construction scheduling problem. A random number generator was developed that could generate a feasible precedence permutation such that an initial population was created for the possible solutions of the scheduling problem. Then, an elitist strategy was adopted that can preserve the best individual generated from the previous generation into the current generation so as to prevent the loss of the best found solutions. Three different termination conditions, that is, number of generations, timeout and number of unique schedules, were used to achieve the finial solution. However, it has been noticed that this method does not work well on large-sized problems. By using the proposed permutation-based elitist GA, Kim and Ellis (2010) compared the efficiencies of two schedule generation schemes, that is, serial scheme and parallel scheme, on decoding the schedule representation into a schedule for resource constrained project. Experimental results demonstrate that the serial scheme is superior to the parallel scheme, which consumes more time for solving each problem.

Chen and Weng (2009) proposed a two-phase GA module for resource constrained project scheduling problem. Concerning the construction constrains, such as precedence relationship, resource requirements and availability, interruption and overlapping of activities, a twophase approach was developed. In the first stage, a GA-based time-cost trade-off analysis was adopted to select the schedule for each activity. Then, a GA-based resource scheduling method was used to generate a feasible solution satisfying the project constraints.

A hybrid GA and simulated annealing module was proposed by Chen and Shahandashti (2009) for solving the multi-project scheduling problem subject to multi-resource constraints. Involvement of the simulated annealing contributes to the selection phase of GA by proposing a new fitness function. As the number of generation increases, the fitness value would increase and induce the algorithm to choose better-fitted solutions. The mutation rate would also decrease as the number of generations grows (Chen and Shahandashti, 2009). The objective of this approach is to minimize the largest finish time of the activities, which does not consider the project cost factors.

On the basis of its random searching mechanism, the GA could solve a variety of optimization problems by searching a larger solution space. Selection of the fitness function is crucial for GA computation. An improperly selected fitness function may cause the algorithm to be trapped into local optimal. It is also difficult for GA to deal with those problems with dynamic data, for example, uncertain construction activities that may be changed frequently. The algorithm may converge to a solution that probably may not work well for the later data. The size of the initial population is also important for the operation of the algorithm. A large population will greatly increase the computation effort, while on the other hand a small population may cause the missing of the optimal solution. Due to its random searching mechanism, GA can always find a better solution compared with the other solutions. However, the solution obtained with GA cannot be 
guaranteed global optimal. As a result, it is difficult to determine the stopping criterion of the algorithm.

Ant colony optimization. Ethnologists have revealed that ants can find the shortest path between their nest and food sources. They discovered that, when ants are searching for food, they lay down pheromones to indicate the path for each other. The pheromone will dissipate over time; however, it increases when other ants travel on the same path. The following ants are willing to choose the path with more pheromones, which leads all ants converging to the same path. ACO is an efficient method for solving combinatorial optimization problems and was founded based on the behaviours of real ants. To implement ACO for solving the construction scheduling problem, we may represent the problem by a weighted network graph. Initial pheromones should be assigned to each edge within the network so as to start the first searching. Then, according to the pheromone information, selection probabilities are determined based on which an artificial ant could travel from the first to the last activity such that the entire project is finished. When the ant travels on the path, pheromones will be updated to the options chosen by the ant to finish the project. Then, a next round iteration starts until the stopping criterion is met.

A number of researchers in construction and engineering have adopted ACO to address time-cost trade-off problems (eg, Ng and Zhang, 2008; Afshar et al, 2009; Lakshminarayanan et al, 2010). The ACO algorithm consists of four elements:

1. Construction solution represents the travelling of an ant through all the activities so as to finish the project.

2. Selection probability determines which node is to be selected based on the pheromone information.

3. Updated pheromone rule memorizes the path when an ant finishes its trip and pheromone will be added to the activities chosen by the ant.

4. A stopping criterion is set to stop the optimization procedure.

$\mathrm{Ng}$ and Zhang (2008) adopted the modified adaptive fitness function, which can be used to evaluate the project time and cost. Two updating rules, that is, a local updating rule and a global updating rule, are presented for the pheromone information updating. Ng and Zhang (2008) experiment demonstrated that the ACO approach can generate a better result than GA by reducing cost with the same duration. When using the ACO approach there is, however, a tendency for premature convergence to occur. Methods for searching the local space adjacent to the solution should be considered so as to obtain a global optimum. Another limitation is that there is no existing criterion for choosing those parameters within the algorithm. Methods that can contribute to selecting the parameters can assist with the implementation of ACO such as neural network and machine learning method.

Afshar et al (2009) developed a non-dominated archiving multi-colony ant algorithm to solve the construction time-cost trade-off optimization problem. A colony of agents is assigned to each objective. Both colonies have the same number of ants and arbitrary objective orders. The solution found in the first colony is transferred to the second colony for evaluation, and the new solution will be transferred back to the first colony for the next iteration cycle. After a number of iterations, the non-dominated solutions are transferred to an external archive where they are compared with each other so as to exclude the dominated solutions. The 'Pareto front' will be obtained after a predetermined number of iterations. Experimental research conducted by Afshar et al (2009) demonstrates that when the number of non-dominated solutions increases, the proposed method can achieve better solutions than the weighting method adopted by the traditional single colony system.

Lakshminarayanan et al (2010) used ACO to solve the extended time-cost-risk trade-off problem of construction scheduling. On the basis of the time-cost trade-off problem, an objective function of the project risk with regard to the utilization of each activity was introduced by using a set of quality indicators. The risk associated with construction project was classified and grouped into a number of zones based on the severity of the risks. The problem was solved by ACO using a test construction project. Similarly, Shrivastava et al (2012) proposed a multi-objective optimization approach for time-costquality-quantity trade-off problem of construction scheduling based on ACO. The objective functions were derived by quantifying the duration, total cost and performance quality. Then, a multi-colony ant system was utilized to solve a test problem introduced by Feng et al (1997). In this multi-objective optimization problem, the weighting parameters chosen by the authors for each objective are 10, 10000 and 0.0005 . However, how to determine those parameters is not specified.

Ant colony is a powerful tool for solving the combinatorial optimization problem. However, several problems should be considered and studied extensively for better applications of this method, for example, the premature convergence phenomenon, the stopping criterion and the parameter determining method.

Particle swarm optimization. PSO is a computational method that can solve the optimization problem by iteratively improving the performance of the solutions according to a given objective measurement. By having a population of candidate particles, PSO can search for the 
optimal solution by moving around its particles in a $D$ dimensional searching space. The position of each particle in the $D$ dimensional space can be expressed by $X_{i}^{t}=$ $\left\{x_{i 1}^{t}, x_{i 2}^{t}, \ldots, x_{i D}^{t}\right\}, i=1,2, \ldots, M, M$ is the population size; $t=1,2, \ldots, T$ represents the generation and $T$ is the iteration limit. Similarly, the particle speed can be expressed by $V_{i}^{t}=\left\{v_{i 1}^{t}, v_{i 2}^{t}, \ldots, v_{i D}^{t}\right\}, i=1,2, \ldots, M$ and $t=1,2, \ldots, T$. The position of each particle is a potential solution to the problem that would be evaluated according to a given objective function. The speed and position of the particles can be updated using the following mathematical formula (Eberhart and Shi, 1998):

$$
\begin{gathered}
v_{i d}^{t}=w v_{i d}^{t-1}+c_{1} \times \operatorname{rand}() \times\left(p_{i d}^{t-1}-x_{i d}^{t-1}\right) \\
+2 \times \operatorname{rand}()\left(p_{g d}^{t}-x_{i d}^{t-1}\right) \\
x_{i d}^{t}=v_{i d}^{t}+x_{i d}^{t-1}
\end{gathered}
$$

where $w$ is an inertia weighting parameter, $\operatorname{rand}()$ represents a random number between 0 and $1, c_{1}$ and $c_{2}$ are positive learning factors, $p_{i d}$ is the local best solution obtained by the $i$ th particle after $t-1$ iterations and $p_{g d}^{t}$ represents the global best solution achieved so far. From (23) and (24), we could see that the particle speed and position are updated iteratively based on the knowledge of the local and global best solutions obtained. As a metaheuristic method, PSO needs few or no assumptions of the problem to be solved and can search a large space for the candidate solutions, which makes it efficient for solving the combinatorial optimization problems.

Zhang et al (2006c) initialized the application of PSO to the CSO problem. A PSO-based approach was proposed to solve the resource constrained project scheduling problem with the objective of minimizing the project duration. To develop a feasible schedule, a particle representation method of activity priorities was adopted that is able to avoid the infeasible sequences from current particle positions. Then, a parallel scheme was used to decode the particles to a feasible schedule according to the precedence and resource constraints. The above two steps form the framework of PSO for solving the resource constrained project scheduling problem. In this study, only one objective, that is, project duration, was considered. While, another important factor, time, was not taken into account.

Zhang et al (2006d) extended the application of PSO to a multi-mode resource constrained project scheduling problem considering both renewable and non-renewable resources. A pair of particle positions was adopted to represent one potential solution by indicating the priority combination and mode combination. Zhang et al (2006b) applied PSO to a preemptive CSP under break and resource constraints with the objective of minimizing the project duration. In this study, preemptive activities that can be interrupted during off-working time (eg, night) were considered. All the resources shared by multi-activities were reallocated during the break. The interrupted activities will not restart immediately after the break due to the resource reallocation. The scheduling priority was represented by multidimensional position of the particle. Then, the problem is solved by PSO and a parallel scheme was adopted to transform the priority to a feasible schedule. In this study, only single mode resources were considered. It will be an interesting future research topic if multiple-mode resource constraints are involved in among different activities.

Guo et al (2010) proposed a modified PSO for solving the CSP for underground mining at the coalface. The modified PSO was developed based on the traditional PSO by introducing a new crossover operator that operates on those coupled particles selected from half of the particle population. The newly created children with better fitness compared with their parents will be chosen for the next iteration. The optimization process consists of two stages. In the first stage, PERT was utilized to derive the time parameters and network graph based on the raw data. In the second stage, the modified PSO was used to optimize the net present value based on the network graph. GA and traditional PSO were also utilized to solve the same problem for comparison. Experimental results demonstrated that the modified PSO is superior to GA and traditional PSO.

PSO is an efficient algorithm for solving the combinatorial optimization problems. However, the solution obtained by PSO is not necessarily a global or local optimum. The selection of the PSO parameters could cause convergence, divergence and oscillation of the particles. To date, PSO parameters are mainly selected based on empirical results. When population variety descends, the particle speed will decrease which, in turn, reduces the capability of the algorithm for searching feasible solutions.

\section{Conclusion and future research}

The CSO has been examined using an array of methods and algorithms. The original single objective optimization problems have been extended to multi-objective trade-off optimization problems subject to various construction constraints. A number of methodologies that have been applied to solve the CSO problem can be classified into three categories: mathematical method, heuristic method and metaheuristic method.

To implement the mathematical method, the problem needs to be explicitly formulated (ie, the objective function and constraints). This is a time-consuming task and difficult for construction planners who do not have the specified mathematical knowledge and background. Some mathematical searching algorithms, for example hill 
climbing algorithm, are single objective oriented and likely to be trapped into local optimality. Therefore, methodologies, by which a global optimality can be obtained, are highly demanded. Constraints are critical factors for solving the CSO problem. Traditional mathematical methods usually treat constraints and objective function separately, that is, optimizing the objective function subject to the constraints. To solve such a problem, we need a feasible point to initiate the searching process and algorithms that can guarantee that the constraints are satisfied.

It is suggested that future research in construction scheduling should consider the application of the exact penalty function method for constrained optimization problems ( $\mathrm{Yu}$ et al, 2010). The method integrates the constraints into the objective function by using several penalty parameters such that the original constrained optimization problem is transformed into an unconstrained optimization problem. It is shown that if the value of the penalty parameter is sufficiently large, then any local minimizer of the corresponding unconstrained optimization problem is a local minimizer of the original problem (Yu et al, 2010). With such a transformation, many existing methods can be utilized to deal with the unconstrained optimization problem, which makes the problem much easier to solve.

The advantage of heuristic methods is their simplicity. Those well-known heuristic methods are Fondahl's method, Structural model method, Siemens approximation method and structural stiffness method. Due to its simplicity and efficiency, Fondahl's method has been adopted by many commercial project scheduling software. However, most heuristic methods are problem dependent, which makes them difficult to be applied to other projects equivalently. It has also been noticed that most of the current heuristic methods focus on single project scheduling. Only a few of them could deal with multiple projects scheduling problem, for example Elazouni (2009), which schedules multi-projects subject to cash constraints. It is suggested that approaches focusing on multiple projects scheduling problems subject to multiobjectives and multi-constraints could be a promising future research direction.

Metaheuristic methods can solve optimization problems by mimicking certain Nature's processes. The most commonly adopted metaheuristic method is the GA for addressing CSO problems. By introducing in the concept of Pareto optimality, a GA can provide a solution candidate pool for the decision maker. Research has focused on how to improve the performance of GA, for example, to prevent the premature convergence and increase the population diversity. In doing so, some enhanced GAs have begun to emerge by modifying the objective weighting parameters, improving the selection, crossover or even the mutation algorithms. However, an efficient and applicable method, which can choose these parameters adaptively, has not been found. As a result it is suggested that artificial intelligence methods such as machine learning and neural networks can evolve their behaviours based on the example data such that they can be used to develop algorithms automatically.

Within the reviewed normative literature, time and cost are commonly considered objectives. Research has focused on minimizing project time and cost so as to achieve maximum profit. However, minimization of time and cost will have an influence on the project quality and risk, which are even more crucial for the successful completion of a construction project. Unfortunately, these key factors have been neglected in most of studies undertaken to date. It is suggested therefore that a multi-objective construction scheduling problem considering both minimization of time-cost-risk and maximization of the quality subject to multiple constraints would be a promising future research topic.

\section{References}

Adeli H and Karim A (1997). Scheduling/cost optimization and neural dynamics model for construction. Journal of Construction Engineering and Management 123(4): 450-458.

Afshar A, Ziaraty AK, Kaveh A and Sgarifi F (2009). Nondominated archiving multicolony ant algorithm in time-cost trade-off optimization. Journal of Construction Engineering and Management 135(7): 668-674.

Antill JM and Woodhead RW (1982). Critical Path Methods in Construction Practices, 3rd edn. Wiley: New York.

Barrie DS and Paulson BC (1984). Professional Construction Management, 2nd edn. McGraw-Hill Publishing Company: New York.

Beasley JE (1996). Advances in Linear and Integer Programming. Oxford Science: Oxford, UK.

Burns SA, Liu L and Feng CW (1996). The LP/IP hybrid method for construction time-cost trade-off analysis. Construction Management and Economics 14(3): 265-276.

Chan WT, Chua DKH and Kannan G (1996). Construction resource scheduling with genetic algorithms. Journal of Construction Engineering and Management 122(2): 125-132.

Chen DS, Batson RG and Dang Y (2010). Applied Integer Programming: Modeling and Solution. John Wiley \& Sons: New York.

Chen PH and Shahandashti SM (2009). Hybrid of genetic algorithm and simulated annealing for multiple project scheduling with multiple resource constraints. Automation in Construction 18(4): 434-443.

Chen PH and Weng H (2009). A two-phase GA model for resourceconstrained project scheduling. Automation in Construction 18(4): 485-498.

Cormican D (1985). Construction Management Planning and Finance. Construction Press: London.

Dasgupta S, Papadimitriou CH and Vazirani UV (2006). Algorithms. McGraw-Hill: New York.

Eberhart RC and Shi Y (1998). Comparison between genetic algorithms and particle swarm optimization. In: Proceedings of the 7th International Conference on Evolutionary Programming VII. San Diego, California, USA, pp 611-616. 
El-Rayes K and Kandil A (2005). Time-cost-quality trade-off analysis for highway construction. ASCE Journal of Construction Engineering and Management 131(4): 477-486.

Elazouni A (2009). Heuristic method for multi-project financebased scheduling. Construction Management and Economics 27(2): 199-211.

Elazouni AM and Gab-Allah AA (2004). Finance-based scheduling of construction projects using integer programming. ASCE Journal of Construction Engineering and Management 130(1): 15-24.

Eshtehardian E, Abbasnia R and Afshar A (2008). Optimization of uncertain construction time-cost trade-off problem. First International Conference on Construction in Developing Countries, August, Karachi, Pakistan.

Fang X (2012). Research of construction schedule optimization using swarm particle optimization. Advanced Materials Research 452/453: 441-445.

Feng CW, Liu L and Burns SA (1997). Using genetic algorithms to solve construction time-cost trade-off problems. ASCE Journal of Construction Engineering and Management 123(3): 184-189.

Fondahl JW (1961). A non-computer approach to the critical path method for the construction industry. Technical Report No. 9, The Construction Institute, Department of Civil Engineering, Stanford University, Stanford, CA.

Fulkerson DR (1961). A network flow computation for project cost curves. Management Science 7(2): 167-178

Galloway PD (2006). Survey of the construction industry relative to the use of CPM scheduling for construction projects. ASCE Journal of Construction Engineering and Management 132(7): 697-711.

Gomar JE, Haas CT and Morton DP (2002). Assignment and allocation optimization of partially multiskilled workforce. ASCE Journal of Construction Engineering and Management 128(2): 103-109.

Guo H, Zhu K, Ding C and Li L (2010). Intelligent optimization for project scheduling of the first mining face in coal mining. Expert Systems with Applications 37(2): 1294-1301.

Hartmann S and Briskorn D (2010). A survey of variants and extensions of the resource-constrained project scheduling problem. European Journal of Operational Research 27(1): 1-14.

Hegazy T (1999a). Optimization of construction time-cost trade-off analysis using genetic algorithms. Canadian Journal of Civil Engineering 26(6): 685-697.

Hegazy T (1999b). Optimization of resource allocation and leveling using genetic algorithms. ASCE Journal of Construction Engineering and Management 125(3): 167-175.

Hegazy T, Shabeeb AK, Elbeitagi E and Cheema T (2000). Algorithm for scheduling with multiskilled construction resources. ASCE Journal of Construction Engineering and Management 126(6): 414-421.

Herroelen W (2005). Project scheduling - theory and practice. Production and Operations Management 14(4): 413-432.

Huang RY and Halpin DW (2000). Graphically based LP modeling for linear scheduling analysis: The POLO system. Engineering, Construction and Architectural Management 7(1): 41-51.

Icmeli O, Erenguc SS and Zappe CJ (1993). Project scheduling problems: A survey. International Journal of Operations and Production Management 3(11): 180-191.

Ipsilandis PG (2006). Multiobjective optimization in linear repetitive project scheduling. Operational Research 6(3): 255-269.

Ipsilandis PG (2007). Multiobjective linear programming model for scheduling linear repetitive projects. ASCE Journal of Construction Engineering and Management 133(6): 417-424.

Jaśkowski P and Sobotka A (2006). Scheduling construction projects using evolutionary algorithms. ASCE Journal of Construction, Engineering and Management 132(8): 861-870.
Jünger M (ed) (2010). 50 Years of Integer Programming 1958-2008: From the Early Years to the State-of-the-Art. Springer-Verlag, New York.

Kallantzis A and Lambropoulos S (2004). Critical path determination by incorporating minimum and maximum time and distance constraints into linear scheduling. Engineering, Construction and Architectural Management 11(3): 211-222.

Kantorovich LV (1940). A new method of solving some classes of extremal problems. Doklady Akad Sci USSR 28: 211-214.

Karlof JK (2005). Integer Programming: Theory and Practice. Taylor \& Francis: Boca Raton, FL.

Kasprowicz T (1994). Multi-objective optimization of construction schedules. In: Proceedings of the 1st Congress on Computing in Civil Engineering, Part 1. Washington DC, pp 185-190.

Kazi AS, Aouad G and Baldwin A (2009). Towards minimizing space-time conflicts between site activities using simple generic algorithm-The best execution strategy. Journal of Information Technology in Construction 14: 154-179.

Kelley JE (1961). Critical-path planning and scheduling: Mathematical basis. Operations Research 9(3): 167-179.

Kelley JE and Walker MR (1959). Critical-path planning and scheduling. In: Proceedings of the Eastern Joint Computer Conference. New York, USA, pp 160-173.

Kim JL and Ellis Jr RD (2008). Permutation-based elitist genetic algorithm for optimization of large-sized resource-constrained project scheduling. ASCE Journal of Construction Engineering and Management 134(11): 904-913.

Kim JL and Ellis Jr RD (2010). Comparing schedule generation schemes in resource-constrained project scheduling using elitist genetic algorithm. ASCE Journal of Construction Engineering and Management 136(2): 160-169.

König M and Beißert U (2009). Construction scheduling optimization using simulated annealing. In: Proceedings of the 26th International Symposium on Automation and Robotics in Construction (ISARC). 24-27 June, Austin, TX.

Lakshminarayanan S, Gaurav A and Arun C (2010). Multiobjective optimization of time-cost-risk using Ant Colony optimization. International Journal of Project Planning and Finance 1(1): 22-38.

Lancaster J and Ozbayrak M (2007). Evolutionary algorithms applied to project scheduling problems - A survey of the stateof-the-art. International Journal of Production Research 45(2): 425-450.

Laptali E, Bouchlagham N and Wild S (1997). Planning and estimating in practice and the use of integrated computer models. Automation in Construction 7(1): 71-76.

Leu SS and Yang CH (1999). GA-based multicriteria optimal model for construction scheduling. Journal of Construction Engineering and Management 125(6): 420-427.

Li H and Love PED (1997). Using improved genetic algorithms to facilitate time-cost optimization. ASCE Journal of Construction Engineering and Management 123(3): 233-237.

Li H, Cao JN and Love PED (1999). Using machine learning and GA to solve time-cost trade-off problems. ASCE Journal of Construction Engineering and Management 125(5): 347-353.

Li H, Love PED and Drew D (2000). Modelling the effects of prolonged overtime work on project cost and quality. Engineering Construction and Architectural Management 7(3): 211-221.

Liu L, Burns SA and Feng CW (1995). Construction time-cost trade-off analysis using LP/IP hybrid method. ASCE Journal of Construction Engineering and Management 121(4): 446-454.

Liu M and Li H (2003). Resource-activity critical-path method for construction planning. ASCE Journal of Construction Engineering and Management 129(4): 412-420. 
Liu SS and Wang CJ (2010). Profit optimization for multiproject scheduling problems considering cash flow. ASCE Journal of Construction Engineering and Management 136(12): 1268-1278.

Long LD and Ohsato A (2009). A genetic algorithm-based method for scheduling repetitive construction projects. Automation in Construction 18(4): 499-511.

Mattila KG and Abraham DM (1998). Resource leveling of linear schedules using integer linear programming. ASCE Journal of Construction Engineering and Management 124(3): 232-244.

Meyer WL and Shaffer LR (1963). Extensions of the critical path method through the application of integer programming. Civil Engineering and Construction Research, Series 2, University of Illinois, Urbana, IL.

Moder JJ, Phillips CR and Davis EW (1983). Project Management with CPM, PERT, and Precedence Diagramming, 3rd edn. Van Nostrand Reinhold Company: New York.

Moselhi O (1993). Schedule compression using the direct stiffness method. Canadian Journal of Civil Engineering 20(1): 65-72.

Moselhi O and El-Rayes K (1993). Scheduling of repetitive projects with cost optimization. ASCE Journal of Construction Engineering and Management 119(4): 681-697.

Ng ST and Zhang Y (2008). Optimizing construction time and cost using ant colony optimization approach. ASCE Journal of Construction Engineering and Management 134(9): 721-728.

Nkasu MM and Leung KH (1997). A resources scheduling decision support system for concurrent project management. International Journal of Production Research 35(11): 3107-3132.

Ozdamar L and Ulusoy G (1995). A survey on the resourceconstrained project scheduling problem. IIE Transactions 27(5): 574-586.

Prager W (1963). A structured method of computing project costpolygons. Management Science 9(3): 394-404.

Que BC (2002). Incorporating practicability into genetic algorithmbased time-cost optimization. ASCE Journal of Construction Engineering and Management 128(2): 139-143.

Robinson DR (1975). A dynamic programming solution to costtime trade-off for CPM. Management Science 22(2): 158-166.

Rogalska M, Bozejko W and Hejducki Z (2008). Time/cost optimization using hybrid evolutionary algorithm in construction project scheduling. Automation in Construction 18(1): 24-31.

Samuel LB (2010). Critical Path Method (CPM). Health Services Policy and Management Courses. University of South Carolina: Columbia, SC.

Senouci AB and Eldin NN (2004). Use of genetic algorithms in resource scheduling of construction projects. ASCE Journal of Construction Engineering and Management 130(6): 869-877.

Shanmuganayagam V (1989). Current float techniques for resources scheduling. ASCE Journal of Construction Engineering and Management 115(3): 401-411.

Shrivastava R, Singh S and Dubey GC (2012). Multi objective optimization of time cost quality quantity using multi colony ant algorithm. International Journal of Contemporary Mathematical Sciences 7(16): 773-784.

Siemens N (1971). A simple CPM time-cost tradeoff algorithm. Management Science 17(6): 354-363.

Sriprasert E and Dawood N (2002). Requirements identification for 4D constraint-based construction planning and control system. International Council for Research and Innovation in Building and Construction-CIB W78 Conference, Construction Informatics
Digital Library, available at http://itc.scix.net/cgi-bin/works/ Show?w78-2002-90.

Sriprasert E and Dawood N (2003). Genetic algorithms for multiconstraint scheduling: An application for the construction industry. In: Proceedings of the 20th CIB W78 Conference on Information Technology in Construction. Auckland, New Zealand, pp 341-352.

Stevens JD (1990). Techniques for Construction Network Scheduling. McGraw-Hill Publishing Company: New York.

Tamimi S and Diekmann J (1988). Soft logic in network analysis. ASCE Journal of Computing in Civil Engineering 2(3): 289-300.

Toklu YC (2002). Application of genetic algorithms to construction scheduling with or without resource constraints. Canadian Journal of Civil Engineering 29(3): 421-429.

Wang $\mathrm{CH}$ and Huang YC (1998). Optimization model for construction project durations using a multistage decision process. Engineering Optimization 30(2): 155-173.

Waugh LM and Froese TM (1991). Constraint knowledge for construction scheduling. First International Conference on Expert Planning Systems, Brighton, UK, pp 114-118.

Willis EM (1986). Scheduling Construction Projects. John Wiley \& Sons: Hoboken, NJ.

Wongwai N and Malaikrisanachalee S (2011). Augmented heuristic algorithm for multi-skilled resource scheduling. Automation in Construction 20(4): 429-445.

Wu IC, Borrmann A, Rank E, Beißert U and Konig M (2009). A pattern-based approach for facilitating schedule generation and cost analysis in bridge construction projects. In: Proceeding of the 26th CIB-W78 Conference on Managing IT in Construction. Istanbul, Turkey, pp 93-100.

Yu C, Teo KL, Zhang LS and Bai YQ (2010). A new exact penalty function method for continuous inequality constrained optimization problems. Journal of Industrial and Management Optimization 6(4): 895-910.

Zhang H, Li H and Tam CM (2006a). Heuristic scheduling of resource-constrained, multiple-mode and repetitive projects. Construction Management and Economics 24(2): 159-169.

Zhang H, Li H and Tam CM (2006b). Particle swarm optimization for preemptive scheduling under break and resource-constraints. ASCE Journal of Construction Engineering and Management 132(3): 259-267.

Zhang H, Li H and Tam CM (2006c). Particle swarm optimization for resource-constrained project scheduling. International Journal of Project Management 24(1): 83-92.

Zhang H, Tam CM and $\mathrm{Li} \mathrm{H}$ (2006d). Multimode project scheduling based on particle swarm optimization. ComputerAided Civil and Infrastructure Engineering 21(2): 93-103.

Zheng DXM, Ng ST and Kumaraswamy MM (2004). Applying a genetic algorithm-based multiobjective approach for time-cost optimization. ASCE Journal of Construction Engineering and Management 130(2): 168-176.

Zheng XM, Ng ST and Kumaraswamy MM (2005). Applying Pareto ranking and niche formation to genetic algorithm-based multiobjective time-cost optimization. ASCE Journal of Construction Engineering and Management 131(1): 81-91.

Received March 2012; accepted November 2012 after one revision 